Abderrahmane El Hachimi (El Jadida)

JAOUAD IGBIDA (El Jadida)

Ahmed Jamea (El Jadida)

\title{
EXISTENCE RESULT FOR NONLINEAR PARABOLIC PROBLEMS WITH $L^{1}$-DATA
}

Abstract. We study the existence of solutions of the nonlinear parabolic problem

$$
\begin{gathered}
\left.\frac{\partial u}{\partial t}-\operatorname{div}\left[|D u-\Theta(u)|^{p-2}(D u-\Theta(u))\right]+\alpha(u)=f \quad \text { in }\right] 0, T[\times \Omega, \\
\left.\left(|D u-\Theta(u)|^{p-2}(D u-\Theta(u))\right) \cdot \eta+\gamma(u)=g \quad \text { on }\right] 0, T[\times \partial \Omega, \\
u(0, \cdot)=u_{0} \quad \text { in } \Omega,
\end{gathered}
$$

with initial data in $L^{1}$. We use a time discretization of the continuous problem by the Euler forward scheme.

1. Introduction. The purpose of this paper is to study the existence question for the following nonlinear parabolic problem

$$
\left\{\begin{array}{l}
\left.\frac{\partial u}{\partial t}-\operatorname{div}(\Phi(D u-\Theta(u)))+\alpha(u)=f \quad \text { in } Q_{T}:=\right] 0, T[\times \Omega \\
\left.\Phi(D u-\Theta(u)) \cdot \eta+\gamma(u)=g \quad \text { on } \Sigma_{T}:=\right] 0, T[\times \partial \Omega \\
u(0, \cdot)=u_{0} \quad \text { in } \Omega
\end{array}\right.
$$

where $\Omega \subset \mathbb{R}^{d}(d \geq 3)$ is an open bounded domain with Lipschitz boundary $\partial \Omega ; T$ is a fixed positive number; $D u$ is the gradient of $u ; \alpha, \gamma, \Theta$ are continuous functions defined on $\mathbb{R}$ and satisfy suitable assumptions; $\eta$ denotes the unit vector normal to $\partial \Omega$; and

$$
\Phi(\xi):=|\xi|^{p-2} \xi, \quad \forall \xi \in \mathbb{R}^{d} .
$$

2010 Mathematics Subject Classification: 35K55, 35K61, 35J60, 35Dxx.

Key words and phrases: entropy solution, nonlinear parabolic problem, Neumann-type boundary conditions, semi-discretization. 
We apply a time discretization of given continuous problem by the Euler forward scheme and study existence, uniqueness and stability questions. We recall that the Euler forward scheme has been used by several authors while studying time discretization of nonlinear parabolic problems; we refer for example to [15, 9, 17, 19] for some details. This scheme is usually used to prove existence of solutions as well as to compute numerical approximations.

The usual weak formulations of parabolic problems in the case where the initial data are in $L^{1}$ do not ensure existence and uniqueness of solutions. New formulations have recently been used, with the hope that a new definition of solution would make it possible to obtain existence and uniqueness. For that, three notions of solution have been adopted: solutions named SOLA (Solution Obtained as the Limit of Approximations) defined by A. Dallaglio [11]; renormalized solutions defined by R. Diperna and P.-L. Lions [14]; and entropy solutions defined by Ph. Bénilan et al. [8]. We will be interested here in the entropy formulation. Many authors have dealt with this type of formulation: see for example [2, 7, 5, 8, 18, 24, 26, 27].

In [18], we considered the case $\Theta=0$ and showed by the same method used here the existence of a unique entropy solution. The same case has been studied by F. Andreu et al. [4]. The problem (1.1) with Dirichlet-type boundary conditions has been studied by many authors: see for example [1, 13, 15, 9, 19, 24].

The problem (1.1), or some special case of it, arises in many different physical contexts. Here we shall mention two of them which are related to turbulent flows.

- Model 1: Filtration of a fluid in a partially saturated porous medium. This flow is governed by the equation

$$
\frac{\partial c(p)}{\partial t}=\nabla a[k(c(p))(\nabla p+e)]
$$

where $p$ is the unknown pressure, $c$ the water content, $k$ the conductivity of the porous medium, $a$ the matrix heterogeneity and $-e$ the direction of gravity. The Kirchhoff transformation equation

$$
u=\int_{0}^{p} k(c(\xi)) d \xi
$$

leads to a differential equation 1.2 of the form

$$
\frac{\partial b(u)}{\partial t}=D a[D u+k(b(u)) e],
$$

where the function $b$ has the same behavior as $c$. 
- Model 2: Flow through a porous medium in a turbulent regime. This model is governed by the continuity equation

$$
\frac{\partial \theta}{\partial t}+\operatorname{div} v=0
$$

and Darcy's law

$$
v=-K(\theta) \operatorname{grad} \phi(\theta),
$$

where $\theta(x, t)$ is the volumetric moisture content, $k(\theta)$ is the hydraulic conductivity, and the total potential $\phi$ is given by

$$
\phi(\theta)=\psi(\theta)+z,
$$

with $\psi(\theta)$ the hydrostatic potential and $z$ the gravitational potential. In turbulent regimes, the flow rate is different from that which can be predicted by the Darcy law, and so several authors proposed a nonlinear relation between $v$ and $K(\theta) \operatorname{grad} \phi$,

$$
|v|^{q-2} v=-K(\theta) \operatorname{grad} \phi(\theta), \quad q>2 .
$$

If $e$ denotes the unit vector in the vertical direction, we obtain

$$
\frac{\partial \theta}{\partial t}-\operatorname{div}\left(|\nabla \varphi(\theta)-K(\theta) e|^{p-2}(\nabla \varphi(\theta)-K(\theta) e)\right)=0,
$$

where

$$
\varphi(\theta)=\int_{0}^{\theta} K(s) \phi^{\prime}(s) d s, \quad p=\frac{q}{q-1} .
$$

This paper is organized as follows: after some preliminary results in Section 2, we discretize the problem (1.1) in Section 3 by the Euler forward scheme and we show the existence and uniqueness of an entropy solution for the discretized problems. Section 4 is devoted to the analysis of the stability of the discretized problems. We finish this paper by studying the existence of an entropy solution to the parabolic problem (1.1).

2. Preliminaries and notations. For a measurable set $\Omega$ in $\mathbb{R}^{d}$, meas $(U)$ denotes its measure, the norm in $L^{p}(\Omega)$ is denoted by $\|\cdot\|_{p}$, and $\|\cdot\|_{1, p}$ denotes the norm in the Sobolev space $W^{1, p}(\Omega) ; C_{i}$ and $C$ will denote various positive constants. For a Banach space $X$ and $a<b, L^{p}(a, b ; X)$ is the space of measurable functions $u:[a, b] \rightarrow X$ such that

$$
\left(\int_{a}^{b}\|u(t)\|_{X}^{p}\right)^{1 / p}=:\|u\|_{L^{p}(a, b ; X)}<\infty .
$$

For a given constant $k>0$ we define the cut-off function $T_{k}: \mathbb{R} \rightarrow \mathbb{R}$ by

$$
T_{k}(s):= \begin{cases}s & \text { if }|s| \leq k \\ k \operatorname{sign}(s) & \text { if }|s|>k\end{cases}
$$


where

$$
\operatorname{sign}(s):= \begin{cases}1 & \text { if } s>0 \\ 0 & \text { if } s=0 \\ -1 & \text { if } s<0 .\end{cases}
$$

For a function $u=u(x), x \in \Omega$, we define the truncated function $T_{k} u$ pointwise, i.e., for every $x \in \Omega$ the value of $T_{k} u$ at $x$ is just $T_{k}(u(x))$.

Define $J_{k}: \mathbb{R} \rightarrow \mathbb{R}^{+}$by

$$
J_{k}(x)=\int_{0}^{x} T_{k}(s) d s
$$

( $J_{k}$ is a primitive of $T_{k}$ ). We have (see [16])

$$
\left\langle\frac{\partial v}{\partial t}, T_{k}(v)\right\rangle=\frac{d}{d t}\left(\int_{\Omega} J_{k}(v)\right) \quad \text { in } L^{1}(] 0, T[),
$$

which implies that

$$
\int_{0}^{t}\left\langle\frac{\partial v}{\partial s}, T_{k}(v)\right\rangle=\int_{\Omega} J_{k}(v(t))-\int_{\Omega} J_{k}(v(0)) .
$$

For $u \in W^{1, p}(\Omega)$, we denote by $\tau u$ or $u$ the trace of $u$ on $\partial \Omega$ in the usual sense. In [8], the authors introduce the following spaces:

$$
\begin{aligned}
& \mathcal{T}_{\text {loc }}^{1,1}(\Omega)=\left\{u: \Omega \rightarrow \mathbb{R} \text { measurable }: T_{k}(u) \in W_{\text {loc }}^{1,1}(\Omega) \text { for all } k>0\right\}, \\
& \mathcal{T}_{\text {loc }}^{1, p}(\Omega)=\left\{u \in \mathcal{T}_{\text {loc }}^{1,1}(\Omega): D T_{k}(u) \in L_{\text {loc }}^{p}(\Omega) \text { for all } k>0\right\}, \\
& \mathcal{T}^{1, p}(\Omega)=\left\{u \in \mathcal{T}_{\text {loc }}^{1, p}(\Omega): D T_{k}(u) \in L^{p}(\Omega) \text { for all } k>0\right\} .
\end{aligned}
$$

For bounded $\Omega$, we have

$$
\mathcal{T}^{1, p}(\Omega)=\left\{u: \Omega \rightarrow \mathbb{R} \text { measurable }: T_{k}(u) \in W^{1, p}(\Omega) \text { for all } k>0\right\} .
$$

Following [8], it is possible to give a meaning to the derivative $D u$ of a function $u \in \mathcal{T}_{\text {loc }}^{1, p}(\Omega)$, generalizing the usual concept of weak derivative in $W_{\text {loc }}^{1,1}(\Omega)$, thanks to the following result:

LEMma $2.1([8])$. For every $u \in \mathcal{T}_{\text {loc }}^{1, p}(\Omega)$ there exists a unique measurable function $v: \Omega \rightarrow \mathbb{R}^{d}$ such that

$$
D T_{k}(u)=v \mathbf{1}_{\{|v|<k\}} \quad \text { a.e. },
$$

where $\mathbf{1}_{B}$ is the characteristic function of the measurable set $B \subset \mathbb{R}^{d}$. Furthermore, $u \in W_{\mathrm{loc}}^{1,1}(\Omega)$ if and only if $v \in L_{\mathrm{loc}}^{1}(\Omega)$, and then $v \equiv D u$ in the usual weak sense.

We also apply the set $\mathcal{T}_{\mathrm{tr}}^{1, p}(\Omega)$ introduced in [4] as the subset of functions in $\mathcal{T}^{1, p}(\Omega)$ for which a generalized notion of trace may be defined. More 
precisely $u \in \mathcal{T}_{\operatorname{tr}}^{1, p}(\Omega)$ if $u \in \mathcal{T}^{1, p}(\Omega)$ and there exist a sequence $\left(u_{n}\right)_{n \in \mathbb{N}}$ in $W^{1, p}(\Omega)$ and a measurable function $v$ on $\partial \Omega$ such that

(a) $u_{n} \rightarrow u$ a.e. in $\Omega$,

(b) $D T_{k}\left(u_{n}\right) \rightarrow D T_{k}(u)$ in $L^{1}(\Omega)$ for every $k>0$,

(c) $u_{n} \rightarrow v$ a.e. on $\partial \Omega$.

The function $v$ is the trace of $u$ in the generalized sense introduced in [4]. For $u \in \mathcal{T}_{\operatorname{tr}}^{1, p}(\Omega)$, the trace of $u$ on $\partial \Omega$ is denoted by $\operatorname{tr}(u)$ or $u$. The operator $\operatorname{tr}(\cdot)$ has the following properties:

(i) if $u \in \mathcal{T}_{\operatorname{tr}}^{1, p}(\Omega)$, then $\tau T_{k}(u)=T_{k}(\operatorname{tr}(u))$ for all $k>0$,

(ii) if $\varphi \in W^{1, p}(\Omega) \cap L^{\infty}(\Omega)$, then for all $u \in \mathcal{T}_{\operatorname{tr}}^{1, p}(\Omega)$, we have $u-\varphi \in$ $\mathcal{T}_{\operatorname{tr}}^{1, p}(\Omega)$ and $\operatorname{tr}(u-\varphi)=\operatorname{tr}(u)-\tau \varphi$.

In the case where $u \in W^{1, p}(\Omega), \operatorname{tr}(u)$ coincides with $\tau u$. Obviously,

$$
W^{1, p}(\Omega) \subset \mathcal{T}_{\operatorname{tr}}^{1, p}(\Omega) \subset \mathcal{T}^{1, p}(\Omega) .
$$

3. The semi-discrete problem. In this section, we discretize the problem (1.1) by the Euler forward scheme and study the questions of existence and uniqueness for the discretized problems. We make the following hypotheses:

$\left(H_{1}\right) \alpha$ and $\gamma$ are nondecreasing continuous real functions on $\mathbb{R}$ such that $\alpha(0)=\gamma(0)=0$;

$\left(H_{2}\right) f \in L^{1}\left(Q_{T}\right), g \in L^{1}\left(\Sigma_{T}\right)$ and $u_{0} \in L^{1}(\Omega)$;

$\left(H_{3}\right) \Theta$ is a continuous function from $\mathbb{R}$ to $\mathbb{R}^{d}$ such that $\Theta(0)=0$ and $|\Theta(x)-\Theta(y)| \leq C|x-y|$ for all $x, y \in \mathbb{R}$, and $C$ is a positive constant such that

$$
C<\frac{(d-p)(\operatorname{meas}(\Omega))^{-1 / d}}{2(d-1) p} .
$$

The Euler forward scheme applied to the problem (1.1) yields the following problems:

$$
\left(\mathcal{P}_{n}\right)\left\{\begin{array}{l}
U^{n}-\tau \operatorname{div}\left(\Phi\left(D U^{n}-\Theta\left(U^{n}\right)\right)\right)+\tau \alpha\left(U^{n}\right)=\tau f_{n}+U^{n-1} \quad \text { in } \Omega, \\
\left(\Phi\left(D U^{n}-\Theta\left(U^{n}\right)\right)\right) \cdot \eta+\gamma\left(U^{n}\right)=g_{n} \quad \text { on } \partial \Omega, \\
U^{0}=u_{0} \quad \text { in } \Omega,
\end{array}\right.
$$

where $N \tau=T, 0<\tau<1,1 \leq n \leq N$ and

$$
\begin{array}{ll}
f_{n}(\cdot)=\frac{1}{\tau} \int_{(n-1) \tau}^{n \tau} f(s, \cdot) d s \quad \text { in } \Omega, \\
g_{n}(\cdot)=\frac{1}{\tau} \int_{(n-1) \tau}^{n \tau} g(s, \cdot) d s & \text { on } \partial \Omega .
\end{array}
$$


Recently, in [8], a new concept of solution has been introduced for the elliptic equation

$$
\begin{aligned}
-\operatorname{div}[\mathbf{a}(x, D u)] & =f(x) & & \text { in } \Omega, \\
u & =0 & & \text { on } \partial \Omega,
\end{aligned}
$$

namely entropy solution. Following this idea we define the concept of entropy solution for the problems $\left(\mathcal{P}_{n}\right)$.

Definition 3.1. An entropy solution to the discretized problems $\left(\mathcal{P}_{n}\right)$ is a sequence $\left(U^{n}\right)_{0 \leq n \leq N}$ such that $U^{0}=u_{0}$ and $U^{n}$ is defined by induction as an entropy solution to the problem

$$
\left\{\begin{array}{l}
u-\tau \operatorname{div}(\Phi(D u-\Theta(u)))+\tau \alpha(u)=\tau f_{n}+U^{n-1} \quad \text { in } \Omega, \\
(\Phi(D u-\Theta(u))) \cdot \eta+\gamma(u)=g_{n} \quad \text { on } \partial \Omega,
\end{array}\right.
$$

i.e. $U^{n} \in \mathcal{T}_{\mathrm{tr}}^{1, p}(\Omega)$ and for all $\varphi \in W^{1, p}(\Omega) \cap L^{\infty}(\Omega)$ and all $k>0$, we have

$$
+\tau \int_{\partial \Omega} \gamma\left(U^{n}\right) T_{k}\left(U^{n}-\varphi\right) \leq \int_{\Omega}\left(\tau f_{n}+U^{n-1}\right) T_{k}\left(U^{n}-\varphi\right)+\tau \int_{\partial \Omega} g_{n} T_{k}\left(U^{n}-\varphi\right) .
$$

Lemma 3.2. Let hypotheses $\left(H_{1}\right)-\left(H_{3}\right)$ be satisfied. If $\left(U^{n}\right)_{0 \leq n \leq N}$ is an entropy solution of problems $\left(\mathcal{P}_{n}\right)$, then $U^{n} \in L^{1}(\Omega)$ for all $n=1, \ldots, N$.

Proof. Taking $\varphi=0$ in (3.1), we get, for $n=1$,

$$
\begin{aligned}
\tau \int_{\Omega} \Phi\left(D U^{1}-\right. & \left.\Theta\left(U^{1}\right)\right) D T_{k}\left(U^{1}\right)+\int_{\Omega}\left(\tau \alpha\left(U^{1}\right)+U^{1}\right) T_{k}\left(U^{1}\right) \\
& +\tau \int_{\partial \Omega} \gamma\left(U^{1}\right) T_{k}\left(U^{1}\right) \leq \int_{\Omega}\left(\tau f_{1}+u_{0}\right) T_{k}\left(U^{1}\right)+\tau \int_{\partial \Omega} g_{1} T_{k}\left(U^{1}\right),
\end{aligned}
$$

i.e.

$$
\begin{aligned}
\tau \int_{\Omega} \Phi\left(D U^{1}-\right. & \left.\Theta\left(U^{1}\right)\right) D T_{k}\left(U^{1}\right)+\int_{\Omega}\left(\tau \alpha\left(U^{1}\right)+U^{1}\right) T_{k}\left(U^{1}\right) \\
& +\frac{\tau}{p} \int_{\Omega}\left|\Theta\left(T_{k}\left(U^{1}\right)\right)\right|^{p} \tau \int_{\partial \Omega} \gamma\left(U^{1}\right) T_{k}\left(U^{1}\right) \\
\leq & \int_{\Omega}\left(\tau f_{1}+u_{0}\right) T_{k}\left(U^{1}\right)+\tau \int_{\partial \Omega} g_{1} T_{k}\left(U^{1}\right)+\frac{\tau}{p} \int_{\Omega}\left|\Theta\left(T_{k}\left(U^{1}\right)\right)\right|^{p} .
\end{aligned}
$$

On the one hand, using the inequality (see [2])

$$
\frac{1}{p}|\xi|^{p}-\frac{1}{p}|\eta|^{p} \leq|\xi|^{p-2} \xi(\xi-\eta), \quad \forall \xi, \eta \in \mathbb{R}^{N} \text { and } 1 \leq p<\infty,
$$


we get

$$
\int_{\Omega} \Phi\left(D U^{1}-\Theta\left(U^{1}\right)\right) D T_{k}\left(U^{1}\right)+\frac{1}{p} \int_{\Omega}\left|\Theta\left(T_{k}\left(U^{1}\right)\right)\right|^{p} \geq 0 .
$$

On the other hand, by assumption $\left(H_{1}\right)$,

$$
\int_{\Omega} \alpha\left(U^{1}\right) T_{k}\left(U^{1}\right)+\int_{\partial \Omega} \gamma\left(U^{1}\right) T_{k}\left(U^{1}\right) \geq 0 .
$$

Therefore, the inequality 3.2 becomes

$$
\int_{\Omega} U^{1} T_{k}\left(U^{1}\right) \leq k \tau\left[\left\|f_{n}\right\|_{1}+\left\|g_{n}\right\|_{L^{1}(\partial \Omega)}\right]+\left\|u_{0}\right\|_{1}+\frac{\tau}{p} \int_{\Omega}\left|\Theta\left(T_{k}\left(U^{1}\right)\right)\right|^{p} .
$$

Since

$$
\sum_{n=1}^{N} \tau\left(\left\|f_{n}\right\|_{1}+\left\|g_{n}\right\|_{L^{1}(\partial \Omega)}\right) \leq\|f\|_{L^{1}\left(Q_{T}\right)}+\|g\|_{L^{1}\left(\Sigma_{T}\right)},
$$

by using hypothesis $\left(H_{3}\right)$ we get

$$
\int_{\Omega} U^{1} T_{k}\left(U^{1}\right) \leq k C_{1}+\frac{\tau}{p}(C k)^{p} \leq k C_{1}+\frac{1}{p}(C k)^{p},
$$

where $C_{1}$ is a constant depending on $f, g, u_{0}$. Since

$$
\lim _{k \rightarrow 0} U^{1} \frac{T_{k}\left(U^{1}\right)}{k}=\left|U^{1}\right|,
$$

dividing (3.4) by $k$ and letting $k \rightarrow 0$, we deduce by Fatou's lemma that

$$
\left\|U^{1}\right\|_{1} \leq C_{2}
$$

THEOREM 3.3. Let hypotheses $\left(H_{1}\right)-\left(H_{3}\right)$ be satisfied and $1<p<d$. Then for all $N \in \mathbb{N}$, the problems $\left(\mathcal{P}_{n}\right)$ have a unique entropy solution $\left(U^{n}\right)_{0 \leq n \leq N}$ such that $U^{n} \in \mathcal{T}_{\operatorname{tr}}^{1, p}(\Omega) \cap L^{1}(\Omega)$ for all $n=1, \ldots, N$.

Proof. Existence. For $n=1$, we can write problem $\left(\mathcal{P}_{1}\right)$ in the form

$$
\begin{aligned}
-\tau \operatorname{div}(\Phi(D u-\Theta(u)))+\bar{\alpha}(u)=F_{1} & \text { in } \Omega, \\
\Phi(D u-\Theta(u)) \eta+\gamma(u)=G_{1} & \text { on } \partial \Omega,
\end{aligned}
$$

where

$$
\bar{\alpha}(s):=\tau \alpha(s)+s, \quad F_{1}:=\tau f+u_{0}, \quad G_{1}:=g .
$$

By hypothesis $\left(H_{2}\right)$, we have $\left(F_{1}, G_{1}\right) \in L^{1}(\Omega) \times L^{1}(\partial \Omega)$, and by hypothesis $\left(H_{1}\right), \bar{\alpha}$ is continuous and $\bar{\alpha}(s) s \geq 0$ for all $s \in \mathbb{R}$. Therefore, using [2, Theorem 3.1], we deduce the existence of an entropy solution $U^{1} \in \mathcal{T}_{\operatorname{tr}}^{1, p}(\Omega)$. 
By induction, using Lemma 3.2, we deduce in the same manner that for $n=1, \ldots, N$, the problem

$$
\begin{aligned}
u-\tau \operatorname{div}(\Phi(D u-\Theta(u)))+\tau \alpha(u) & =\tau f_{n}+U^{n-1} & & \text { in } \Omega, \\
\Phi(D u-\Theta(u)) \eta+\gamma(u) & =g_{n} & & \text { on } \partial \Omega,
\end{aligned}
$$

has an entropy solution $U^{n}$ in $\mathcal{T}_{\mathrm{tr}}^{1, p}(\Omega)$.

Uniqueness. We first need the following lemma.

Lemma 3.4. For all $n=1, \ldots, N$ we have

(i) $\lim _{h \rightarrow \infty} \lim _{k \rightarrow 0} \frac{1}{k} \int_{\left\{h<\left|U^{n}\right|<k+h\right\}} \Phi\left(D U^{n}-\Theta\left(U^{n}\right)\right) D U^{n}=0$,

(ii) $\lim _{h \rightarrow \infty} \lim _{k \rightarrow 0} \frac{1}{k} \int_{\left\{h<\left|U^{n}\right|<k+h\right\}}\left|D U^{n}\right|^{p}=0$,

(iii) $\lim _{h \rightarrow \infty} \lim _{k \rightarrow 0} \frac{1}{k} \int_{0}^{t} \int_{\left\{h<\left|U^{n}\right|<k+h\right\}}\left|D U^{n}-\Theta\left(U^{n}\right)\right|^{p}=0$.

Proof. Let $n=1$; for simplicity, we write $u=U^{1}$.

For (i), taking $\varphi=T_{h}(u)$ as a test function, we get

$$
\begin{aligned}
& \tau \int_{\Omega} \Phi(D u-\Theta(u)) D T_{k}\left(u-T_{h}(u)\right)+\int_{\Omega}(\tau \alpha(u)+u) T_{k}\left(u-T_{h}(u)\right) \\
& +\int_{\partial \Omega} \tau \gamma(u) T_{k}\left(u-T_{h}(u)\right) \leq \int_{\Omega}\left(\tau f_{1}+u_{0}\right) T_{k}\left(u-T_{h}(u)\right)+\int_{\partial \Omega} \tau g_{1} T_{k}\left(u-T_{h}(u)\right) .
\end{aligned}
$$

Dividing by $k$ and taking the limit as $k \rightarrow 0$ and $h \rightarrow \infty$, we obtain

$$
\begin{aligned}
\lim _{h \rightarrow \infty} \lim _{k \rightarrow 0} \frac{1}{k} \int_{\{h<|u|<k+h\}} \Phi(D u-\Theta(u)) D u & \\
& \leq \lim _{h \rightarrow \infty} \lim _{k \rightarrow 0}\left(\int_{\{|u|>h\}}\left(\tau\left|f_{1}\right|+\left|u_{0}\right|\right)+\int_{\partial \Omega \cap\{|u|>h\}} \tau\left|g_{1}\right|\right),
\end{aligned}
$$

i.e.

$$
\lim _{h \rightarrow \infty} \lim _{k \rightarrow 0} \frac{1}{k} \int_{\{h<|u|<k+h\}} \Phi(D u-\Theta(u)) D u=0,
$$

which proves (i).

Applying (3.3) and (i), we easily get (ii) and (iii).

Let $\left(U^{n}\right)_{0 \leq n \leq N}$ and $\left(V^{n}\right)_{0 \leq n \leq N}, N \in \mathbb{N}$, be two entropy solutions of problems $\left(\mathcal{P}_{n}\right)$ and let $\varphi \in W^{\overline{1}, p}(\Omega) \cap L^{\infty}(\Omega)$. For $n=1$ we get (for sim- 
plicity, we write $u=U^{1}, v=V^{1}$ )

$$
\begin{aligned}
\tau \int_{\Omega} \Phi(D u & -\Theta(u)) D T_{k}(u-\varphi)+\int_{\Omega}(\tau \alpha(u)+u) T_{k}(u-\varphi) \\
& +\int_{\partial \Omega} \tau \gamma(u) T_{k}(u-\varphi) \leq \int_{\Omega}\left(\tau f_{1}+u_{0}\right) T_{k}(u-\varphi)+\int_{\partial \Omega} \tau g_{1} T_{k}(u-\varphi),
\end{aligned}
$$

and

$$
\begin{aligned}
\tau \int_{\Omega} \Phi(D v- & \Theta(v)) D T_{k}(v-\varphi)+\int_{\Omega}(\tau \alpha(v)+v) T_{k}(v-\varphi) \\
& +\int_{\partial \Omega} \tau \gamma(v) T_{k}(v-\varphi) \leq \int_{\Omega}\left(\tau f_{1}+u_{0}\right) T_{k}(v-\varphi)+\int_{\partial \Omega} \tau g_{1} T_{k}(v-\varphi) .
\end{aligned}
$$

Let $h>0$. For the solution $u$ we take $\varphi=T_{h}(v)$ and for the solution $v$ we take $\varphi=T_{h}(u)$ as test functions; summing the above inequalities and letting $k \rightarrow 0, h \rightarrow \infty$, using hypothesis $\left(H_{1}\right)$, we obtain

$$
\tau \lim _{h \rightarrow \infty} \lim _{k \rightarrow 0} \frac{1}{k} \mathcal{I} \mathcal{I}(k, h)+\|u-v\|_{1} \leq 0,
$$

where

$\mathcal{I} \mathcal{I}(k, h)=\int_{\Omega} \Phi(D u-\Theta(u)) D T_{k}\left(u-T_{h}(v)\right)+\int_{\Omega} \Phi(D v-\Theta(v)) D T_{k}\left(v-T_{h}(u)\right)$.

Now, we consider the following decomposition:

$$
\begin{array}{ll}
\Omega_{1}(h)=\{|u| \leq h,|v| \leq h\}, & \Omega_{2}(h)=\{|u| \leq h,|v|>h\}, \\
\Omega_{3}(h)=\{|u|>h,|v| \leq h\}, & \Omega_{2}(h)=\{|u|>h,|v|>h\},
\end{array}
$$

and

$$
\mathcal{I} \mathcal{I}(k, h)=\sum_{i=1}^{4} \mathcal{L}_{i}(k, h)
$$

where

$$
\mathcal{L}_{i}(k, h)=\int_{\Omega_{i}(h)}\left[\Phi(D v-\Theta(v)) D T_{k}\left(v-T_{h}(u)\right)+\Phi(D u-\Theta(u)) D T_{k}\left(u-T_{h}(v)\right)\right] .
$$

We have

$$
\begin{aligned}
\mathcal{L}_{1}(k, h) & =\int_{\Omega_{1}(h)} \Phi(D v-\Theta(v)) D T_{k}(v-u)+\int_{\Omega_{1}(h)} \Phi(D u-\Theta(u)) D T_{k}(u-v) \\
& =\mathcal{L}_{1}^{1}(k, h)+\mathcal{L}_{1}^{2}(k, h),
\end{aligned}
$$

where

$$
\mathcal{L}_{1}^{1}(k, h)=\int_{\Omega_{1}(h, k)}[\Phi(D v-\Theta(v))-\Phi(D u-\Theta(u))(D v-\Theta(v)-D u+\Theta(u))],
$$




$$
\mathcal{L}_{1}^{2}(k, h)=\int_{\Omega_{1}(h, k)}[\Phi(D v-\Theta(v))-\Phi(D u-\Theta(u))(\Theta(v)-\Theta(u))],
$$

where

$$
\Omega_{1}(h, k)=\{|u| \leq h,|v| \leq h,|v-u| \leq k\} .
$$

First, we show that

$$
\lim _{h \rightarrow \infty} \lim _{k \rightarrow 0} \frac{1}{k} \mathcal{L}_{1}(k, h) \geq 0 .
$$

CASE $1<p \leq 2$. Let $\varepsilon>0$. By Young's inequality, we have

$$
\begin{aligned}
\left|\mathcal{L}_{1}^{2}(k, h)\right| \leq & \frac{\varepsilon}{p^{\prime}} \int_{\Omega_{1}(h, k)}|\Phi(D v-\Theta(v))-\Phi(D u-\Theta(u))|^{p^{\prime}} \\
& +\frac{1}{p \varepsilon} \int_{\Omega_{1}(h, k)}|\Theta(v)-\Theta(u)|^{p} .
\end{aligned}
$$

Using hypothesis $\left(H_{3}\right)$ and the inequality (see [13])

$|\Phi(\xi)-\Phi(\eta)|^{p^{\prime}} \leq C\{(\xi-\eta)(\Phi(\xi)-\Phi(\eta))\}^{\beta / 2}\left\{|\xi|^{p}+|\eta|^{p}\right\}^{1-\beta / 2}, \forall \xi, \eta \in \mathbb{R}^{d}$, where $\beta=2$ if $1<p \leq 2$ and $\beta=p^{\prime}$ if $p \geq 2$, we obtain

which implies

$$
\left|\mathcal{L}_{1}^{2}(k, h)\right| \leq \frac{\varepsilon C_{1}}{p^{\prime}} \mathcal{L}_{1}^{1}(k, h)+\frac{C_{2}}{p \varepsilon} k^{p},
$$

$$
\left|\lim _{k \rightarrow 0} \frac{1}{k} \mathcal{L}_{1}^{2}(k, h)\right| \leq \frac{\varepsilon C_{1}}{p^{\prime}} \lim _{k \rightarrow 0} \frac{1}{k} \mathcal{L}_{1}^{1}(k, h) .
$$

If $\lim _{k \rightarrow 0} \frac{1}{k} \mathcal{L}_{1}^{1}(k, h)=+\infty$, we use Hölder's inequality to get

$$
\begin{aligned}
\left|\mathcal{L}_{1}^{2}(k, h)\right| & \\
\leq\left[\left(\int_{\Omega_{1}(h, k)}|D v-\Theta(v)|^{p}\right)^{1 / p^{\prime}}+\right. & \left.\left(\int_{\Omega_{1}(h, k)}|D u-\Theta(u)|^{p}\right)^{1 / p^{\prime}}\right] \\
& \times\left(\int_{\Omega_{1}(h, k)}|\Theta(v)-\Theta(u)|^{p}\right)^{1 / p} .
\end{aligned}
$$

On the other hand, taking $\varphi=0$ in (3.1), we get

$$
\int_{\{|u| \leq k\}}|D u-\Theta(u)|^{p} \leq k C_{3}, \quad \forall k>0 .
$$

Then, by hypothesis $\left(H_{3}\right)$, inequality (3.6) becomes

$$
\left|\mathcal{L}_{1}^{2}(k, h)\right| \leq C_{4}(h+k)^{1 / p^{\prime}} k .
$$

This implies that

$$
\lim _{k \rightarrow 0} \frac{1}{k} \mathcal{L}_{1}^{1}(k, h)+\lim _{k \rightarrow 0} \frac{1}{k} \mathcal{L}_{1}^{2}(k, h)=+\infty,
$$


SO

$$
\lim _{h \rightarrow \infty} \lim _{k \rightarrow 0} \frac{1}{k} \mathcal{L}_{1}(k, h)=+\infty .
$$

If $\lim _{k \rightarrow 0} \frac{1}{k} \mathcal{L}_{1}^{1}(k, h)=0$, using $(3.5)$, we obtain $\lim _{k \rightarrow 0} \frac{1}{k} \mathcal{L}_{1}^{2}(k, h)=0$. Hence

$$
\lim _{h \rightarrow \infty} \lim _{k \rightarrow 0} \frac{1}{k} \mathcal{L}_{1}(k, h)=0 .
$$

If $0<\lim _{k \rightarrow 0} \frac{1}{k} \mathcal{L}_{1}^{1}(k, h)<\infty$, we take

$$
\varepsilon=\frac{1}{h \lim _{k \rightarrow 0} \frac{1}{k} \mathcal{L}_{1}^{1}(k, h)}
$$

in (3.5) to deduce that

$$
\lim _{h \rightarrow \infty} \lim _{k \rightarrow 0} \frac{1}{k} \mathcal{L}_{1}^{2}(k, h)=0,
$$

and it follows that

$$
\lim _{h \rightarrow \infty} \lim _{k \rightarrow 0} \frac{1}{k} \mathcal{L}_{1}(k, h) \geq 0 .
$$

CASE $p>2$. By using Young's inequality, we obtain

$$
\frac{1}{k}\left|\mathcal{L}_{1}^{2}(k, h)\right| \leq \frac{C_{5} \varepsilon(k+h)}{p^{\prime} k}+\frac{C_{6}}{p \varepsilon} k^{p-1}, \quad \forall \varepsilon>0 .
$$

Taking $\varepsilon=k / h^{2}$, we get

$$
\lim _{h \rightarrow \infty} \lim _{k \rightarrow 0} \frac{1}{k} \mathcal{L}_{1}^{2}(k, h)=0 .
$$

It follows that

$$
\lim _{h \rightarrow \infty} \lim _{k \rightarrow 0} \frac{1}{k} \mathcal{L}_{1}(k, h) \geq 0
$$

Now, we have

$$
\begin{aligned}
\mathcal{L}_{2}(k, h)= & \int_{\Omega_{2}(h)} \Phi(D v-\Theta(v)) D T_{k}(v-u) \\
& +\int_{\Omega_{2}(h)} \Phi(D u-\Theta(u)) D T_{k}(u-h \operatorname{sign}(v)) \\
= & \mathcal{L}_{2}^{2}(k, h)+\mathcal{L}_{2}^{1}(k, h)
\end{aligned}
$$

where

$$
\begin{aligned}
\mathcal{L}_{2}^{2}(k, h): & =\int_{\Omega_{2}(h)} \Phi(D u-\Theta(u)) D T_{k}(u-h \operatorname{sign}(v)) \\
& =\int_{\Omega_{2}^{1}(k, h)} \Phi(D u-\Theta(u)) D u \geq 0 .
\end{aligned}
$$


And

$$
\begin{aligned}
\mathcal{L}_{2}^{1}(k, h) & :=\int_{\Omega_{2}(h)} \Phi(D v-\Theta(v)) D T_{k}(v-u) \\
& =\int_{\Omega_{2}^{2}(k, h)} \Phi(D v-\Theta(v)) D v-\int_{\Omega_{2}^{2}(k, h)} \Phi(D v-\Theta(v)) D u
\end{aligned}
$$

where

$$
\begin{aligned}
& \Omega_{2}^{1}(k, h)=\Omega_{2}(h) \cap\{|u-h \operatorname{sign}(v)| \leq k\}, \\
& \Omega_{2}^{2}(k, h)=\{|u| \leq h:|v|>h,|v-u| \leq k\} .
\end{aligned}
$$

By hypothesis $\left(H_{3}\right)$,

$$
\int_{\Omega_{2}^{2}(k, h)} \Phi(D v-\Theta(v)) D v \geq 0 .
$$

By using Hölder's inequality, we get

$$
\left|\int_{\Omega_{2}^{2}(k, h)} \Phi(D v-\Theta(v)) D u\right| \leq\left(\int_{\Omega_{2}^{2}(k, h)}|D v-\Theta(v)|^{p}\right)^{1 / p^{\prime}}\left(\int_{\Omega_{2}^{2}(k, h)}|D u|^{p}\right)^{1 / p}
$$

where $1 / p+1 / p^{\prime}=1$. Thus, by applying Lemma 3.4 , we obtain

$$
\lim _{h \rightarrow \infty} \lim _{k \rightarrow 0} \frac{1}{k} \int_{\Omega_{2}^{2}(k, h)} \Phi(D v-\Theta(v)) D u=0 .
$$

Then

$$
\lim _{h \rightarrow \infty} \lim _{k \rightarrow 0} \frac{1}{k} \mathcal{L}_{2}^{1}(k, h) \geq 0,
$$

In the same manner, we show that

$$
\lim _{h \rightarrow \infty} \lim _{k \rightarrow 0} \frac{1}{k}\left(\mathcal{L}_{3}(k, h)+\mathcal{L}_{4}(k, h)\right) \geq 0 .
$$

Thus

$$
\lim _{h \rightarrow \infty} \lim _{k \rightarrow 0} \frac{1}{k} \mathcal{I} \mathcal{I}(k, h) \geq 0 .
$$

This implies that $\|u-v\|_{1} \leq 0$. By induction we prove that

$$
\forall n=1, \ldots, N, \quad U^{n}=V^{n},
$$

which gives the uniqueness of solution of problems $\left(\mathcal{P}_{n}\right)$.

4. Stability. In this section, we give some a priori estimates for the discrete entropy solution $\left(U^{n}\right)_{1 \leq n \leq N}$ which we use later to derive convergence results for the Euler forward scheme.

THeOREM 4.1. Let hypotheses $\left(H_{1}\right)-\left(H_{3}\right)$ be satisfied and $1<p<d$. Then there exists a positive constant $C\left(u_{0}, f, g\right)$ depending on the data but 
not on $N$ such that for all $n=1, \ldots, N$, we have

1. $\left\|U^{n}\right\|_{1} \leq C\left(u_{0}, f, g\right)$,

2. $\tau \sum_{i=1}^{n}\left\|\alpha\left(U^{i}\right)\right\|_{1}+\tau \sum_{i=1}^{n}\left\|\gamma\left(U^{i}\right)\right\|_{L^{1}(\partial \Omega)} \leq C\left(u_{0}, f, g\right)$,

3. $\sum_{i=1}^{n}\left\|U^{i}-U^{i-1}\right\|_{1} \leq C\left(u_{0}, f, g\right)$

4. $\sum_{i=1}^{n} \tau\left\|T_{k}\left(U^{i}\right)\right\|_{1, p}^{p} \leq k C\left(u_{0}, f, g\right)$.

Proof. $1 \& 2$ : Taking $\varphi=0$ as a test function in (3.1) and dividing this inequality by $k$, we obtain

$$
\begin{aligned}
\tau \int_{\Omega}\left(\Phi \left(D T_{k}\left(U^{i}\right)-\Theta\right.\right. & \left.\left.\left(T_{k}\left(U^{i}\right)\right)\right) D T_{k}\left(U^{i}\right)+\frac{1}{p}\left|\Theta\left(T_{k}\left(U^{i}\right)\right)\right|^{p}\right) \\
& +\int_{\Omega} U^{i} \frac{T_{k}\left(U^{i}\right)}{k}+\tau \int_{\Omega} \alpha\left(U^{i}\right) \frac{T_{k}\left(U^{i}\right)}{k}+\tau \int_{\partial \Omega} \gamma\left(U^{i}\right) \frac{T_{k}\left(U^{i}\right)}{k} \\
\leq & \tau\left(\left\|f_{i}\right\|_{1}+\left\|g_{i}\right\|_{L^{1}(\partial \Omega)}\right)+\left\|U^{i-1}\right\|_{1}+\frac{1}{k p}\left\|\Theta\left(T_{k}\left(U^{i}\right)\right)\right\|_{p}^{p},
\end{aligned}
$$

i.e.

$$
\begin{aligned}
\int_{\Omega} U^{i} \frac{T_{k}\left(U^{i}\right)}{k}+ & \tau \int_{\Omega} \alpha\left(U^{i}\right) \frac{T_{k}\left(U^{i}\right)}{k}+\tau \int_{\partial \Omega} \gamma\left(U^{i}\right) \frac{T_{k}\left(U^{i}\right)}{k} \\
& \leq \tau\left(\left\|f_{i}\right\|_{1}+\left\|g_{i}\right\|_{L^{1}(\partial \Omega)}\right)+\left\|U^{i-1}\right\|_{1}+\frac{1}{k p}\left\|\Theta\left(T_{k}\left(U^{i}\right)\right)\right\|_{p}^{p} .
\end{aligned}
$$

Using hypothesis $\left(H_{3}\right)$ and letting $k \rightarrow 0$, we deduce by Fatou's lemma that

$$
\begin{aligned}
\left\|U^{i}\right\|_{1}+\tau\left\|\alpha\left(U^{i}\right)\right\|_{1}+\tau\left\|\gamma\left(U^{i}\right)\right\|_{L^{1}(\partial \Omega)} & \\
& \leq \tau\left(\left\|f_{i}\right\|_{1}+\left\|g_{i}\right\|_{L^{1}(\partial \Omega)}\right)+\left\|U^{i-1}\right\|_{1} .
\end{aligned}
$$

Summing (4.1) from $i=1$ to $n$ we obtain

$$
\begin{aligned}
\left\|U^{n}\right\|_{1}+\tau \sum_{i=1}^{n}\left\|\alpha\left(U^{i}\right)\right\|_{1}+\tau \sum_{i=1}^{n} \| & \gamma\left(U^{i}\right) \|_{L^{1}(\partial \Omega)} \\
& \leq\|f\|_{L^{1}\left(Q_{T}\right)}+\|g\|_{L^{1}\left(\Sigma_{T}\right)}+\left\|u_{0}\right\|_{1} .
\end{aligned}
$$

This implies inequalities 1 and 2 .

3: Taking $\varphi=T_{h}\left(U^{i}-\operatorname{sign}\left(U^{i}-U^{i-1}\right)\right)(h>1)$ as a test function in (3.1) and letting $h \rightarrow \infty$, we get, for $k \geq 1$, 


$$
\begin{aligned}
\tau \lim _{h \rightarrow \infty} \mathcal{I}(k, h)+\left\|U^{i}-U^{i-1}\right\|_{1} \\
\quad \leq \tau\left[\left\|f_{i}\right\|_{1}+\left\|g_{i}\right\|_{L^{1}(\partial \Omega)}+\left\|\alpha\left(U^{i}\right)\right\|_{1}+\left\|\gamma\left(U^{i}\right)\right\|_{L^{1}(\partial \Omega)}\right]
\end{aligned}
$$

where

$$
\begin{aligned}
\mathcal{I}(k, h) & :=\int_{\Omega} \Phi\left(D U^{i}-\Theta\left(U^{i}\right)\right) D T_{k}\left(U^{i}-T_{h}\left(U^{i}-\operatorname{sign}\left(U^{i}-U^{i-1}\right)\right)\right), \\
& =\int_{\Omega(k, h) \cap \bar{\Omega}(k)} \Phi\left(D U^{i}-\Theta\left(U^{i}\right)\right) D\left(U^{i}\right),
\end{aligned}
$$

and

$$
\begin{aligned}
\Omega(k, h) & :=\left\{\left|U^{i}-T_{h}\left(U^{i}-\operatorname{sign}\left(U^{i}-U^{i-1}\right)\right)\right| \leq k\right\}, \\
\bar{\Omega}(k) & :=\left\{\left|U^{i}-\operatorname{sign}\left(U^{i}-U^{i-1}\right)\right|>h\right\} .
\end{aligned}
$$

As

$$
\Omega(k, h) \cap \bar{\Omega}(k) \subset\left\{k-1 \leq\left|U^{i}\right| \leq k+h\right\},
$$

we conclude by using [2, Lemma 3.6] that

$$
\lim _{h \rightarrow \infty} \mathcal{I}(k, h)=0 .
$$

Thus, inequality 4.2 becomes

(4.3) $\left\|U^{i}-U^{i-1}\right\|_{1} \leq \tau\left[\left\|f_{i}\right\|_{1}+\left\|g_{i}\right\|_{L^{1}(\partial \Omega)}+\left\|\alpha\left(U^{i}\right)\right\|_{1}+\left\|\gamma\left(U^{i}\right)\right\|_{L^{1}(\partial \Omega)}\right]$.

Summing (4.3) from $i=1$ to $n$ and using the stability result 2, we obtain the stability result 3 .

4: In (3.1), we take $\varphi=0$ as a test function and use hypothesis $\left(H_{1}\right)$ to get

$$
\tau \int_{\Omega} \Phi\left(D U^{i}-\Theta\left(U^{i}\right)\right) D T_{k}\left(U^{i}\right) \leq k \tau\left[\left\|f_{i}\right\|_{1}+\left\|g_{i}\right\|_{L^{1}(\partial \Omega)}\right]+k\left\|U^{i}-U^{i-1}\right\|_{1} .
$$

Using the inequalities

$$
\begin{array}{rlrl}
(a+b)^{p} & \leq 2^{p-1}\left(a^{p}+b^{p}\right), & \forall a, b & \in \mathbb{R}^{+} \text {and } 1 \leq p \leq \infty, \\
\frac{1}{p}|\xi|^{p}-\frac{1}{p}|\eta|^{p} & \leq|\xi|^{p-2} \xi(\xi-\eta), & \forall \xi, \eta \in \mathbb{R}^{N} \text { and } 1 \leq p<\infty,
\end{array}
$$

and hypothesis $\left(H_{3}\right)$, we deduce that

$$
\tau\left\|D T_{k}\left(U^{i}\right)\right\|_{p}^{p} \leq k \tau\left[\left\|f_{i}\right\|_{1}+\left\|g_{i}\right\|_{L^{1}(\partial \Omega)}\right]+k\left\|U^{i}-U^{i-1}\right\|_{1} .
$$

Now, summing (4.4) from $i=1$ to $n$ and using the stability result 3 , we get

$$
\sum_{i=1}^{n} \tau\left\|T_{k}\left(U^{i}\right)\right\|_{1, p}^{p} \leq k C\left(u_{0}, f, g\right)
$$


5. Convergence and existence result. In this section, using the above results, we build an entropy solution of problem (1.1). We start by giving the entropy formulation of the nonlinear parabolic problem (1.1).

Definition 5.1. A measurable function $u: Q_{T} \rightarrow \mathbb{R}$ is an entropy solution of the parabolic problem (1.1) in $Q_{T}$ if

$$
u \in C\left(0, T ; L^{1}(\Omega)\right), \quad T_{k}(u) \in L^{p}\left(0, T ; W^{1, p}(\Omega)\right)
$$

and for all $k>0$ we have

$$
\begin{aligned}
\int_{0}^{t} \int_{\Omega} \Phi(D & u-\Theta(u)) D T_{k}(u-\varphi)+\int_{0}^{t} \int_{\Omega} \alpha(u) T_{k}(u-\varphi)+\int_{0}^{t} \int_{\partial \Omega} \gamma(u) T_{k}(u-\varphi) \\
\leq & -\int_{0}^{t}\left\langle\frac{\partial \varphi}{\partial s}, T_{k}(u-\varphi)\right\rangle+\int_{\Omega} J_{k}(u(0)-\varphi(0))-\int_{\Omega} J_{k}(u(t)-\varphi(t)) \\
& +\int_{0}^{t} \int_{\Omega} f T_{k}(u-\varphi)+\int_{0}^{t} \int_{\partial \Omega} g T_{k}(u-\varphi)
\end{aligned}
$$

for all $\varphi \in L^{\infty}\left(Q_{T}\right) \cap L^{p}\left(0, T ; W^{1, p}(\Omega)\right) \cap W^{1,1}\left(0, T ; L^{1}(\Omega)\right)$ and $t \in[0, T]$.

Now, we state our main result.

Theorem 5.2. Let hypotheses $\left(H_{1}\right)-\left(H_{3}\right)$ be satisfied and $1<p<d$. Then the nonlinear parabolic problem (1.1) has an entropy solution.

Proof. Let us introduce a piecewise linear extension (called the Rothe function)

$$
\left\{\begin{array}{l}
u^{N}(0):=u_{0}, \\
u^{N}(t):=U^{n-1}+\left(U^{n}-U^{n-1}\right) \frac{t-t^{n-1}}{\tau}, \\
\left.\forall t \in] t^{n-1}, t^{n}\right], n=1, \ldots, N, \text { in } \Omega,
\end{array}\right.
$$

and a piecewise constant function

$$
\left\{\begin{array}{l}
\bar{u}^{N}(0):=u_{0}, \\
\left.\left.\bar{u}^{N}(t):=U^{n}, \quad \forall t \in\right] t^{n-1}, t^{n}\right], n=1, \ldots, N, \text { in } \Omega,
\end{array}\right.
$$

where $t^{n}:=n \tau$.

By Theorem 3.3, for any $N \in \mathbb{N}$, the solution $\left(U^{n}\right)_{1 \leq n \leq N}$ of problems $\left(\mathcal{P}_{n}\right)$ is unique. Thus, $u^{N}$ and $\bar{u}^{N}$ are uniquely defined. And by using the stability results of Theorem 4.1, we deduce the existence of a constant $C\left(T, u_{0}, f, g\right)$ not depending on $N$ such that for all $N \in \mathbb{N}$, we have 


$$
\begin{aligned}
& \left\|\bar{u}^{N}-u^{N}\right\|_{L^{1}\left(Q_{T}\right)} \leq \frac{1}{N} C\left(T, u_{0}, f, g\right), \\
& \left\|u^{N}\right\|_{L^{1}\left(Q_{T}\right)} \leq C\left(T, u_{0}, f, g\right), \\
& \left\|\bar{u}^{N}\right\|_{L^{1}\left(Q_{T}\right)} \leq C\left(T, u_{0}, f, g\right), \\
& \left\|\frac{\partial u^{N}}{\partial t}\right\|_{L^{1}\left(Q_{T}\right)} \leq C\left(T, u_{0}, f, g\right), \\
& \left\|T_{k}\left(\bar{u}^{N}\right)\right\|_{L^{p}\left(0, T ; W^{1, p}(\Omega)\right)} \leq k C\left(T, u_{0}, f, g\right), \\
& \left\|\alpha\left(\bar{u}^{N}\right)\right\|_{L^{1}\left(Q_{T}\right)} \leq C\left(T, u_{0}, f, g\right), \\
& \left\|\gamma\left(\bar{u}^{N}\right)\right\|_{L^{1}\left(\Sigma_{T}\right)} \leq C\left(T, u_{0}, f, g\right) .
\end{aligned}
$$

Lemma 5.3. Let hypotheses $\left(H_{1}\right)-\left(H_{3}\right)$ be satisfied and $1<p<d$. Then the sequence $\left(\bar{u}^{N}\right)_{N \in \mathbb{N}}$ converges in measure and a.e. in $Q_{T}$.

Proof. Let $\varepsilon, r, k$ be positive numbers. For $N, M \in \mathbb{N}$, we have the inclusion

$$
\begin{aligned}
\left\{\left|\bar{u}^{N}-\bar{u}^{M}\right|>r\right\} \subset & \left\{\left|\bar{u}^{N}\right|>k\right\} \cup\left\{\left|\bar{u}^{M}\right|>k\right\} \\
& \cup\left\{\left|\bar{u}^{N}\right| \leq k,\left|\bar{u}^{M}\right| \leq k,\left|\bar{u}^{N}-\bar{u}^{M}\right|>r\right\} .
\end{aligned}
$$

On the one hand

$$
\operatorname{meas}\left\{\left|\bar{u}^{N}\right|>k\right\} \leq \frac{1}{k}\left\|\bar{u}^{N}\right\|_{L^{1}\left(Q_{T}\right)} \leq \frac{1}{k} C\left(T, u_{0}, f, g\right) .
$$

In the same manner we have

$$
\operatorname{meas}\left\{\left|\bar{u}^{M}\right|>k\right\} \leq \frac{1}{k} C\left(T, u_{0}, f, g\right) .
$$

Thus, for a sufficiently large $k$, we have

$$
\operatorname{meas}\left(\left\{\left|\bar{u}^{N}\right|>k\right\} \cup\left\{\left|\bar{u}^{M}\right|>k\right\}\right) \leq \varepsilon / 2 \text {. }
$$

On the other hand, by (5.3) we deduce that the sequence $\left(T_{k}\left(\bar{u}^{N}\right)_{N \in \mathbb{N}}\right)$ is bounded in $L^{p}\left(Q_{T}\right)$. Hence, there exists a subsequence, still denoted by $\left(T_{k}\left(\bar{u}^{N}\right)\right)_{N \in \mathbb{N}}$, that is a Cauchy sequence in $L^{p}\left(Q_{T}\right)$ and in measure. Thus, there exists $N_{0} \in \mathbb{N}$ such that for all $N, M \geq N_{0}$, we have

$$
\operatorname{meas}\left(\left\{\left|\bar{u}^{N}\right| \leq k,\left|\bar{u}^{M}\right| \leq k,\left|\bar{u}^{N}-\bar{u}^{M}\right|>r\right\}\right)<\varepsilon / 2 .
$$

Then, by (5.4) and (5.5), $\left(\bar{u}^{N}\right)_{N \in \mathbb{N}}$ converges in measure. Therefore there exists an element $u \in M\left(Q_{T}\right)$ such that

$$
\bar{u}^{N} \rightarrow u \quad \text { a.e. in } Q_{T} \text {. }
$$

Now, by 5.3 ,

$$
\left(D T_{k}\left(\bar{u}^{N}\right)\right)_{N \in \mathbb{N}} \text { is uniformly bounded in } L^{p}\left(Q_{T}\right) \text {. }
$$

Hence there exists a subsequence, still denoted by $\left(D T_{k}\left(\bar{u}^{N}\right)\right)_{N \in \mathbb{N}}$, such that $\left(D T_{k}\left(\bar{u}^{N}\right)\right)_{N \in \mathbb{N}}$ converges to an element $V$ in $L^{p}\left(Q_{T}\right)$. 
But

$$
T_{k}\left(\bar{u}^{N}\right) \text { converges to } T_{k}(u) \text { in } L^{p}\left(Q_{T}\right) .
$$

Hence,

$$
D T_{k}\left(\bar{u}^{N}\right) \text { converges to } D T_{k}(u) \text { weakly in } L^{p}\left(Q_{T}\right),
$$

and by $(5.3)$ we conclude that

$$
T_{k}(u) \in L^{p}\left(0, T ; W^{1, p}(\Omega)\right) \quad \text { for all } k>0 .
$$

We now prove that $\left(\bar{u}^{N}\right)_{N \in \mathbb{N}}$ converges a.e. in $\Sigma_{T}$. Set

$$
A_{k}=\left\{(t, x) \in \Sigma_{T}:\left|T_{k}(u(t))\right|<k\right\}, \quad \forall k>0 .
$$

Since the operator trace $\tau: W^{1, p}(\Omega) \rightarrow L^{p}(\partial \Omega)$ is compact, there is a constant $C>0$ such that

$$
\int_{0}^{T}\left\|T_{k}\left(\bar{u}^{N}(t)\right)-T_{k}(u(t))\right\|_{L^{1}(\partial \Omega)} \leq C \int_{0}^{T}\left\|T_{k}\left(\bar{u}^{N}(t)\right)-T_{k}(u(t))\right\|_{W^{1,1}(\partial \Omega)} .
$$

But $T_{k}\left(\bar{u}^{N}\right) \rightarrow T_{k}(u)$ in $L^{1}\left(\Sigma_{T}\right)$; hence by a diagonal process, we can find a subsequence $\bar{u}^{N_{j}}$ and a null subset $D \subset \Sigma_{T}$ such that

$$
\lim _{j \rightarrow \infty}\left(T_{k}\left(\bar{u}^{N_{j}}(t)\right)\right)(x)=\left(T_{k}(u(t))\right)(x) \quad \text { for all }(t, x) \in \Sigma_{T} \backslash D .
$$

This implies that

$$
\lim _{j \rightarrow \infty} \bar{u}^{N_{j}}(t, x)=v(t, x) \quad \text { for all }(t, x) \in \bigcup_{k=1}^{\infty} A_{k} \backslash D,
$$

where

$$
v(t, x)=\left(T_{k}(u(t))\right)(x) \quad \text { if }(t, x) \in A_{k} .
$$

Lemma 5.4. The sequence $\left(u^{N}\right)_{N \in \mathbb{N}}$ converges to $u$ in $C\left(0, T ; L^{1}(\Omega)\right)$.

Proof. Let $\left(t^{n}=n \tau_{N}\right)_{n=1}^{N}$ and $\left(t^{m}=m \tau_{M}\right)_{m=1}^{M}$ be two partitions of the interval $[0, T]$ and let $\left(u^{N}(t), \bar{u}^{N}(t)\right),\left(u^{M}(t), \bar{u}^{M}(t)\right)$ be the semi-discrete solutions defined by (5.1), (5.2) and corresponding to the respective partitions. Let $\varphi \in L^{\infty}\left(Q_{T}\right) \cap L^{p}\left(0, T ; W^{1, p}(\Omega)\right) \cap W^{1,1}\left(0, T ; L^{1}(\Omega)\right)$ and $t \in[0, T]$. We rewrite (3.1) in the forms

$$
\begin{aligned}
\int_{0}^{t}\left\langle\frac{\partial u^{N}}{\partial s}, T_{k}\left(\bar{u}^{N}-\varphi\right)\right\rangle+\int_{0}^{t} \int_{\Omega} \Phi\left(D \bar{u}^{N}-\Theta\left(\bar{u}^{N}\right)\right) D T_{k}\left(\bar{u}^{N}-\varphi\right) \\
\quad+\int_{0}^{t} \int_{\Omega} \alpha\left(\bar{u}^{N}\right) T_{k}\left(\bar{u}^{N}-\varphi\right)+\int_{0}^{t} \int_{\partial \Omega} \gamma\left(\bar{u}^{N}\right) T_{k}\left(\bar{u}^{N}-\varphi\right) \\
\leq \int_{0}^{t} \int_{\Omega} f_{N} T_{k}\left(\bar{u}^{N}-\varphi\right)+\int_{0}^{t} \int_{\partial \Omega} g_{N} T_{k}\left(\bar{u}^{N}-\varphi\right)
\end{aligned}
$$


and

$$
\begin{aligned}
\int_{0}^{t}\left\langle\frac{\partial u^{M}}{\partial s}, T_{k}\left(\bar{u}^{M}-\varphi\right)\right\rangle+\int_{0}^{t} \int_{\Omega} \Phi\left(D \bar{u}^{M}-\Theta\left(\bar{u}^{M}\right)\right) D T_{k}\left(\bar{u}^{M}-\varphi\right) \\
\quad+\int_{0}^{t} \int_{\Omega} \alpha\left(\bar{u}^{M}\right) T_{k}\left(\bar{u}^{M}-\varphi\right)+\int_{0}^{t} \int_{\partial \Omega} \gamma\left(\bar{u}^{M}\right) T_{k}\left(\bar{u}^{M}-\varphi\right) \\
\leq \int_{0}^{t} \int_{\Omega} f_{M} T_{k}\left(\bar{u}^{M}-\varphi\right)+\int_{0}^{t} \int_{\partial \Omega} g_{M} T_{k}\left(\bar{u}^{M}-\varphi\right),
\end{aligned}
$$

where

$$
\begin{array}{lll}
f_{N}(t, x)=f_{n}(x), & g_{N}(t, x)=g_{n}(x) & \left.\forall t \in] t^{n-1}, t^{n}\right], n=1, \ldots, N . \\
f_{M}(t, x)=f_{m}(x), & g_{M}(t, x)=g_{m}(x) & \left.\forall t \in] t^{m-1}, t^{m}\right], m=1, \ldots, M .
\end{array}
$$

Let $h>1$. In inequality (5.6) we take $\varphi=T_{k}\left(u^{M}\right)$ and in inequality (5.7) we take $\varphi=T_{k}\left(u^{N}\right)$. Summing both inequalities, we get, for $k=1$,

$$
\begin{aligned}
& \int_{0}^{t}\left\langle\frac{\partial\left(u^{N}-u^{M}\right)}{\partial s}, T_{1}\left(u^{N}-u^{M}\right)\right\rangle+I_{N, M}(h)+\int_{0}^{t} \int_{\Omega} \alpha\left(\bar{u}^{N}\right) T_{1}\left(\bar{u}^{N}-T_{h}\left(\bar{u}^{M}\right)\right) \\
& \quad+\int_{0}^{t} \int_{\Omega} \alpha\left(\bar{u}^{M}\right) T_{1}\left(\bar{u}^{M}-T_{h}\left(\bar{u}^{N}\right)\right) \\
& \quad+\int_{0}^{t} \int_{\partial \Omega}\left[\gamma\left(\bar{u}^{N}\right) T_{1}\left(\bar{u}^{N}-T_{h}\left(\bar{u}^{M}\right)\right)+\gamma\left(\bar{u}^{M}\right) T_{1}\left(\bar{u}^{M}-T_{h}\left(\bar{u}^{N}\right)\right)\right] \\
& \leq \int_{0}^{t}\left\langle\frac{\partial\left(u^{N}-u^{M}\right)}{\partial s}, T_{1}\left(u^{N}-u^{M}\right)\right\rangle-\left\langle\frac{\partial u^{N}}{\partial s}, T_{1}\left(\bar{u}^{N}-T_{h}\left(\bar{u}^{M}\right)\right)\right\rangle \\
& \quad-\int_{0}^{t}\left\langle\frac{\partial u^{M}}{\partial s}, T_{1}\left(\bar{u}^{M}-T_{h}\left(\bar{u}^{N}\right)\right)\right\rangle \\
& \quad+\int_{0}^{t} \int_{\Omega}\left[f_{N} T_{1}\left(\bar{u}^{N}-T_{h}\left(\bar{u}^{M}\right)\right)+f_{M} T_{1}\left(\bar{u}^{M}-T_{h}\left(\bar{u}^{N}\right)\right)\right] \\
& \quad+\int_{0}^{t} \int_{\partial \Omega}\left[g_{N} T_{1}\left(\bar{u}^{N}-T_{h}\left(\bar{u}^{M}\right)\right)+g_{M} T_{1}\left(\bar{u}^{M}-T_{h}\left(\bar{u}^{N}\right)\right)\right],
\end{aligned}
$$

where

$$
\begin{aligned}
I_{N, M}(h)= & \int_{0}^{t} \int_{\Omega} \Phi\left(D \bar{u}^{N}-\Theta\left(\bar{u}^{N}\right)\right) D T_{1}\left(\bar{u}^{N}-T_{h}\left(\bar{u}^{M}\right)\right) \\
& +\int_{0}^{t} \int_{\Omega} \Phi\left(D \bar{u}^{M}-\Theta\left(\bar{u}^{M}\right)\right) D T_{1}\left(\bar{u}^{M}-T_{h}\left(\bar{u}^{N}\right)\right) .
\end{aligned}
$$


We have

$$
\begin{aligned}
\left|\int_{0}^{t}\left\langle\frac{\partial\left(u^{N}-u^{M}\right)}{\partial s}, T_{1}\left(u^{N}-u^{M}\right)\right\rangle\right| & \\
\leq & \left\|\frac{\partial\left(u^{N}-u^{M}\right)}{\partial s}\right\|_{L^{1}\left(Q_{T}\right)}\left\|T_{1}\left(u^{N}-u^{M}\right)\right\|_{L^{\infty}\left(Q_{T}\right)} \\
& \leq 2 C\left(T, f, g, u_{0}\right)\left\|T_{1}\left(u^{N}-u^{M}\right)\right\|_{L^{\infty}\left(Q_{T}\right)} .
\end{aligned}
$$

Since

$$
\lim _{N, M \rightarrow \infty}\left\|T_{1}\left(u^{N}-u^{M}\right)\right\|_{L^{\infty}\left(Q_{T}\right)}=0
$$

we deduce that

$$
\lim _{h \rightarrow \infty} \lim _{N, M \rightarrow \infty} \int_{0}^{t}\left\langle\frac{\partial\left(u^{N}-u^{M}\right)}{\partial s}, T_{1}\left(u^{N}-u^{M}\right)\right\rangle=0 .
$$

Similarly, we show that

$$
\begin{gathered}
\lim _{h \rightarrow \infty} \lim _{N, M \rightarrow \infty}\left(\int_{0}^{t}\left\langle\frac{\partial u^{N}}{\partial s}, T_{1}\left(\bar{u}^{N}-T_{h}\left(\bar{u}^{M}\right)\right)\right\rangle+\int_{0}^{t}\left\langle\frac{\partial u^{M}}{\partial s}, T_{1}\left(\bar{u}^{M}-T_{h}\left(\bar{u}^{N}\right)\right)\right\rangle\right)=0, \\
\lim _{h \rightarrow \infty} \lim _{N, M \rightarrow \infty} \int_{0}^{t} \int_{\Omega}\left[f_{N} T_{1}\left(\bar{u}^{N}-T_{h}\left(\bar{u}^{M}\right)\right)+f_{M} T_{1}\left(\bar{u}^{M}-T_{h}\left(\bar{u}^{N}\right)\right)\right]=0, \\
\lim _{h \rightarrow \infty, M \rightarrow \infty} \lim _{0} \int_{\partial \Omega}^{t}\left[g_{N} T_{1}\left(\bar{u}^{N}-T_{h}\left(\bar{u}^{M}\right)\right)+g_{M} T_{1}\left(\bar{u}^{M}-T_{h}\left(\bar{u}^{N}\right)\right)\right]=0,
\end{gathered}
$$

and

$$
\begin{gathered}
\lim _{h \rightarrow \infty} \lim _{N, M \rightarrow \infty} \int_{0}^{t} \int_{\Omega} \alpha\left(\bar{u}^{N}\right) T_{1}\left(\bar{u}^{N}-T_{h}\left(\bar{u}^{M}\right)\right)+\int_{0} \int_{\Omega} \alpha\left(\bar{u}^{M}\right) T_{1}\left(\bar{u}^{M}-T_{h}\left(\bar{u}^{N}\right)\right)=0, \\
\lim _{N, M \rightarrow \infty} \int_{0}^{t} \int_{\partial \Omega}\left[\gamma\left(\bar{u}^{N}\right) T_{1}\left(\bar{u}^{N}-T_{h}\left(\bar{u}^{M}\right)\right)+\gamma\left(\bar{u}^{M}\right) T_{1}\left(\bar{u}^{M}-T_{h}\left(\bar{u}^{N}\right)\right)\right]=0 .
\end{gathered}
$$

Then, letting $N, M \rightarrow \infty$ and $h \rightarrow \infty$, we get

$$
\lim _{h \rightarrow \infty} \lim _{N, M \rightarrow \infty} \int_{0}^{t}\left\langle\frac{\partial\left(u^{N}-u^{M}\right)}{\partial s}, T_{1}\left(u^{N}-u^{M}\right)\right\rangle+\lim _{h \rightarrow \infty} \lim _{M \rightarrow \infty} I_{N, M}(h) \leq 0 .
$$

Since

$$
\left\langle\frac{\partial v}{\partial t}, T_{k}(v)\right\rangle=\frac{d}{d t} \int_{\Omega} J_{k}(v) \quad \text { in } L^{1}(] 0, T[)
$$


A. El Hachimi et al.

inequality (5.9) becomes

$$
\lim _{N, M \rightarrow \infty} \int_{\Omega} J_{1}\left(u^{N}(t)-u^{M}(t)\right)+\lim _{h \rightarrow \infty} \lim _{N, M \rightarrow \infty} I_{N, M}(h) \leq 0 .
$$

Now, we show that

$$
\lim _{h \rightarrow \infty} \lim _{N, M \rightarrow \infty} I_{N, M}(h) \geq 0 .
$$

We consider the decomposition

$$
I_{N, M}(h)=\sum_{i=1}^{4} L_{i}(h),
$$

where

$$
\begin{aligned}
L_{i}(h)= & \int_{0}^{t} \int_{\Omega_{i}(h)} \Phi\left(D \bar{u}^{N}-\Theta\left(\bar{u}^{N}\right)\right) D T_{1}\left(\bar{u}^{N}-T_{h}\left(\bar{u}^{M}\right)\right) \\
& +\int_{0}^{t} \int_{\Omega_{i}(h)} \Phi\left(D \bar{u}^{M}-\Theta\left(\bar{u}^{M}\right)\right) D T_{1}\left(\bar{u}^{M}-T_{h}\left(\bar{u}^{N}\right)\right),
\end{aligned}
$$

and

$$
\begin{array}{ll}
\Omega_{1}(h)=\left\{\left|\bar{u}^{N}\right| \leq h,\left|\bar{u}^{M}\right| \leq h\right\}, & \Omega_{2}(h)=\left\{\left|\bar{u}^{N}\right| \leq h,\left|\bar{u}^{M}\right|>h\right\}, \\
\Omega_{3}(h)=\left\{\left|\bar{u}^{N}\right|>h,\left|\bar{u}^{M}\right| \leq h\right\}, & \Omega_{4}(h)=\left\{\left|\bar{u}^{N}\right|>h,\left|\bar{u}^{M}\right|>h\right\} .
\end{array}
$$

On the one hand, we have

$$
\begin{aligned}
& L_{1}(h)=\int_{0}^{t} \int_{\Omega_{1}^{1}(h)}\left[\Phi\left(D \bar{u}^{N}-\Theta\left(\bar{u}^{N}\right)\right)-\Phi\left(D \bar{u}^{M}-\Theta\left(\bar{u}^{M}\right)\right)\right] D\left(\bar{u}^{N}-\bar{u}^{M}\right) \\
& =\int_{0}^{t} \int_{\Omega_{1}^{1}(h)}\left[\Phi\left(D \bar{u}^{N}-\Theta\left(\bar{u}^{N}\right)\right)-\Phi\left(D \bar{u}^{M}-\Theta\left(\bar{u}^{M}\right)\right)\right] \Psi_{\Theta}\left(\bar{u}^{N}, \bar{u}^{M}\right) \\
& \quad+\int_{0}^{t} \int_{\Omega_{1}^{1}(h)}\left[\Phi\left(D \bar{u}^{N}-\Theta\left(\bar{u}^{N}\right)\right)-\Phi\left(D \bar{u}^{M}-\Theta\left(\bar{u}^{M}\right)\right)\right]\left(\Theta\left(\bar{u}^{N}\right)-\Theta\left(\bar{u}^{M}\right)\right) \\
& \geq \int_{0 \Omega_{1}^{1}(h)}^{t}\left[\Phi\left(D T_{h}\left(\bar{u}^{N}\right)-\Theta\left(T_{h}\left(\bar{u}^{N}\right)\right)\right)-\Phi\left(D T_{h}\left(\bar{u}^{M}\right)-\Theta\left(T_{h}\left(\bar{u}^{M}\right)\right)\right)\right] \Lambda_{\Theta}^{h}\left(\bar{u}^{N}, \bar{u}^{M}\right),
\end{aligned}
$$

where

$$
\Psi_{\Theta}\left(\bar{u}^{N}, \bar{u}^{M}\right)=D \bar{u}^{N}-\Theta\left(\bar{u}^{N}\right)-\left(D \bar{u}^{M}-\Theta\left(\bar{u}^{M}\right)\right),
$$

and

$$
\begin{gathered}
\Lambda_{\Theta}^{h}\left(\bar{u}^{N}, \bar{u}^{M}\right)=\Theta\left(T_{h}\left(\bar{u}^{N}\right)\right)-\Theta\left(T_{h}\left(\bar{u}^{M}\right)\right), \\
\Omega_{1}^{1}(h)=\left\{\left|\bar{u}^{N}\right| \leq h,\left|\bar{u}^{M}\right| \leq h,\left|\bar{u}^{M}-\bar{u}^{M}\right| \leq 1\right\} .
\end{gathered}
$$


Since

$$
\Theta\left(T_{h}\left(\bar{u}^{N}\right)\right)-\Theta\left(T_{h}\left(\bar{u}^{M}\right)\right) \rightarrow 0 \quad \text { strongly in } L^{p}\left(Q_{T}\right)
$$

and

$$
\Phi\left(D T_{h}\left(\bar{u}^{N}\right)-\Theta\left(T_{h}\left(\bar{u}^{M}\right)\right)\right)-\Phi\left(D T_{h}\left(\bar{u}^{N}\right)-\Theta\left(T_{h}\left(\bar{u}^{M}\right)\right)\right)
$$

converges weakly in $L^{p^{\prime}}\left(Q_{T}\right)$, it follows that the integral

$\int_{0}^{t} \int_{\Omega_{1}(k)}\left[\Phi\left(D T_{h}\left(\bar{u}^{N}\right)-\Theta\left(T_{h}\left(\bar{u}^{N}\right)\right)\right)-\Phi\left(D T_{h}\left(\bar{u}^{M}\right)-\Theta\left(T_{h}\left(\bar{u}^{M}\right)\right)\right)\right] \Lambda_{\Theta}^{h}\left(\bar{u}^{N}, \bar{u}^{M}\right)$

tends to zero. Therefore

$$
\lim _{h \rightarrow \infty} \lim _{N, M \rightarrow \infty} L_{1}(h) \geq 0 .
$$

On the other hand, we have

$$
\begin{aligned}
L_{2}(h)= & \int_{0}^{t} \int_{\Omega_{2}^{1}(h)} \Phi\left(D \bar{u}^{N}-\Theta\left(\bar{u}^{N}\right)\right) D \bar{u}^{N} \\
& +\int_{0}^{t} \int_{\Omega_{2}^{2}(h)} \Phi\left(D \bar{u}^{M}-\Theta\left(\bar{u}^{M}\right)\right) D\left(\bar{u}^{M}-\bar{u}^{N}\right) \\
\geq & -\int_{0}^{t} \int_{\Omega_{2}^{2}(h)} \Phi\left(D \bar{u}^{M}-\Theta\left(\bar{u}^{M}\right)\right) D \bar{u}^{N},
\end{aligned}
$$

where

$$
\begin{aligned}
& \Omega_{2}^{1}(h)=\left\{\left|\bar{u}^{N}\right| \leq h,\left|\bar{u}^{M}\right|>h,\left|\bar{u}^{N}-h \operatorname{sign}\left(\bar{u}^{M}\right)\right| \leq 1\right\}, \\
& \Omega_{2}^{2}(h)=\left\{\left|\bar{u}^{N}\right| \leq h,\left|\bar{u}^{M}\right|>h,\left|\bar{u}^{N}-\bar{u}^{M}\right| \leq 1\right\} .
\end{aligned}
$$

Now, taking $\varphi=T_{h}\left(\bar{u}^{N}\right)$ in $(5.6)$, we deduce that

$$
\lim _{h \rightarrow \infty} \lim _{N \rightarrow \infty} \int_{0}^{t} \int_{\left\{h \leq\left|\bar{u}^{N}\right| \leq h+k\right\}} \Phi\left(D \bar{u}^{N}-\Theta\left(\bar{u}^{N}\right)\right) D \bar{u}^{N}=0, \quad \forall k>0 .
$$

This implies

$$
\begin{aligned}
& \lim _{h \rightarrow \infty} \lim _{N \rightarrow \infty} \int_{0}^{t} \int_{\left\{h \leq\left|\bar{u}^{N}\right| \leq h+k\right\}}\left|D \bar{u}^{N}-\Theta\left(\bar{u}^{N}\right)\right|^{p}=0, \forall k>0, \\
& \lim _{h \rightarrow \infty} \lim _{N \rightarrow \infty} \int_{0}^{t} \int_{\left\{h \leq\left|\bar{u}^{N}\right| \leq h+k\right\}}\left|D \bar{u}^{N}\right|^{p}=0, \quad \forall k>0 .
\end{aligned}
$$


Now,

$$
\begin{aligned}
& \left|\int_{0}^{t} \int_{\Omega_{2}^{2}(h)} \Phi\left(D \bar{u}^{M}-\Theta\left(\bar{u}^{M}\right)\right) D \bar{u}^{N}\right| \\
& \quad \leq\left(\int_{0}^{t} \int_{\left\{h \leq\left|\bar{u}^{M}\right| \leq h+1\right\}}\left|D \bar{u}^{M}-\Theta\left(\bar{u}^{M}\right)\right|^{p}\right)^{1 / p^{\prime}}\left(\int_{0}^{t} \int_{\left\{h-1 \leq\left|\bar{u}^{N}\right| \leq h\right\}}\left|D \bar{u}^{N}\right|^{p}\right)^{1 / p} .
\end{aligned}
$$

Thus 5.11 and 5.12 give

$$
\lim _{N, M \rightarrow \infty} \int_{0}^{t} \int_{\Omega_{2}^{2}(h)} \Phi\left(D \bar{u}^{M}-\Theta\left(\bar{u}^{M}\right)\right) D \bar{u}^{N}=0,
$$

which implies that

$$
\lim _{h \rightarrow \infty} \lim _{N, M \rightarrow \infty} L_{2}(h) \geq 0 .
$$

Similarly, we show that

$$
\lim _{h \rightarrow \infty} \lim _{N, M \rightarrow \infty}\left(L_{3}(k, h)+L_{4}(k, h)\right) \geq 0 .
$$

Therefore

$$
\lim _{h \rightarrow \infty} \lim _{N \rightarrow \infty} I(h) \geq 0 .
$$

Thus 5.10 becomes

$$
\lim _{N, M \rightarrow \infty} \int_{\Omega} J_{1}\left(u^{N}(t)-u^{M}(t)\right)=0 .
$$

Since

$$
\begin{array}{r}
\frac{1}{2} \int_{\left\{\left|u^{N}-u^{M}\right| \leq 1\right\}}\left|u^{N}(t)-u^{M}(t)\right|^{2}+\int_{\left\{\left|u^{N}-u^{M}\right| \geq 1\right\}}\left|u^{N}(t)-u^{M}(t)\right| \\
\leq \int_{\Omega} J_{1}\left(u^{N}(t)-u^{M}(t)\right),
\end{array}
$$

we have

$$
\begin{aligned}
& \int_{\Omega}\left|u^{N}(t)-u^{M}(t)\right| \\
& =\int_{\left\{\left|u^{N}-u^{M}\right| \leq 1\right\}}\left|u^{N}(t)-u^{M}(t)\right|+\int_{\left\{\left|u^{N}-u^{M}\right| \geq 1\right\}}\left|u^{N}(t)-u^{M}(t)\right| \\
& \leq C_{1}(\Omega)\left(\int_{\left\{\left|u^{N}-u^{M}\right| \leq 1\right\}}\left|u^{N}(t)-u^{M}(t)\right|^{2}\right)^{1 / 2}+\int_{\left\{\left|u^{N}-u^{M}\right| \geq 1\right\}}\left|u^{N}(t)-u^{M}(t)\right| \\
& \leq C_{2}(\Omega)\left(\int_{\Omega} J_{1}\left(u^{N}(t)-u^{M}(t)\right)\right)^{1 / 2}+\int_{\Omega} J_{1}\left(u^{N}(t)-u^{M}(t)\right) .
\end{aligned}
$$


By (5.13), we deduce that $\left(u^{N}\right)_{N \in \mathbb{N}}$ is a Cauchy sequence in $C\left(0, T ; L^{1}(\Omega)\right)$. Hence $\left(u^{N}\right)_{N \in \mathbb{N}}$ converges to $u$ in $C\left(0, T ; L^{1}(\Omega)\right)$.

Now, we prove that the limit function $u$ is an entropy solution of the problem (1.1). Since $u^{N}(0)=U^{0}=u_{0}$ for all $N \in \mathbb{N}$, we have $u(0, \cdot)=u_{0}$, and inequality $(5.6)$ implies

$$
\begin{aligned}
& \int_{0}^{t}\left\langle\frac{\partial u^{N}}{\partial s}, T_{k}\left(\bar{u}^{N}-\varphi\right)-T_{k}\left(u^{N}-\varphi\right)\right\rangle+\int_{0}^{t} \int_{\Omega} \Phi\left(D \bar{u}^{N}-\Theta\left(\bar{u}^{N}\right)\right) D T_{k}\left(\bar{u}^{N}-\varphi\right) \\
& \quad+\int_{0}^{t} \int_{\Omega} \alpha\left(\bar{u}^{N}\right) T_{k}\left(\bar{u}^{N}-\varphi\right)+\int_{0}^{t} \int_{\partial \Omega} \gamma\left(\bar{u}^{N}\right) T_{k}\left(\bar{u}^{N}-\varphi\right) \\
& \leq-\int_{0}^{t}\left\langle\frac{\partial \varphi}{\partial s}, T_{k}\left(u^{N}-\varphi\right)\right\rangle+\int_{\Omega} J_{k}\left(u^{N}(0)-\varphi(0)\right)-\int_{\Omega} J_{k}\left(u^{N}(t)-\varphi(t)\right) \\
& \quad+\int_{0}^{t} \int_{\Omega} f_{N} T_{k}\left(\bar{u}^{N}-\varphi\right)+\int_{0}^{t} \int_{\partial \Omega} g_{N} T_{k}\left(\bar{u}^{N}-\varphi\right) .
\end{aligned}
$$

Let $\bar{k}=k+\|\varphi\|_{\infty}$. Then

$$
\begin{aligned}
\int_{0}^{t} \int_{\Omega} \Phi\left(D \bar{u}^{N}-\Theta\left(\bar{u}^{N}\right)\right) D T_{k}\left(\bar{u}^{N}-\varphi\right) & \\
= & \int_{0}^{t} \int_{\Omega} \Phi\left(D T_{\bar{k}}\left(\bar{u}^{N}\right)-\Theta\left(T_{\bar{k}}\left(\bar{u}^{N}\right)\right)\right) D T_{k}\left(T_{\bar{k}}\left(\bar{u}^{N}\right)-\varphi\right) \\
= & \int_{0}^{t} \int_{\Omega}\left[\Phi\left(D T_{\bar{k}}\left(\bar{u}^{N}\right)-\Theta\left(T_{\bar{k}}\left(\bar{u}^{N}\right)\right)\right) D T_{\bar{k}}\left(\bar{u}^{N}\right)\right. \\
& \left.-\Phi\left(D T_{\bar{k}}\left(\bar{u}^{N}\right)-\Theta\left(T_{\bar{k}}\left(\bar{u}^{N}\right)\right)\right) D \varphi\right] \mathbf{1}_{Q(N, k)},
\end{aligned}
$$

where $Q(N, k)=\left\{\left|T_{\bar{k}}\left(\bar{u}^{N}\right)-\varphi\right| \leq k\right\}$.

Thus, the inequality (5.14) becomes

$$
\begin{aligned}
& \int_{0}^{t}\left\langle\frac{\partial u^{N}}{\partial s}, T_{k}\left(\bar{u}^{N}-\varphi\right)-T_{k}\left(u^{N}-\varphi\right)\right\rangle \\
& -\int_{0}^{t} \int_{\Omega} \Phi\left(D T_{\bar{k}}\left(\bar{u}^{N}\right)-\Theta\left(T_{\bar{k}}\left(\bar{u}^{N}\right)\right)\right) D \varphi \mathbf{1}_{Q(N, k)} \\
& +\int_{0}^{t} \int_{\Omega}\left[\Phi\left(D T_{\bar{k}}\left(\bar{u}^{N}\right)-\Theta\left(T_{\bar{k}}\left(\bar{u}^{N}\right)\right)\right) D T_{\bar{k}}\left(\bar{u}^{N}\right)+\frac{1}{p}\left|\Theta\left(T_{\bar{k}}\left(\bar{u}^{N}\right)\right)\right|^{p}\right] \mathbf{1}_{Q(N, k)}
\end{aligned}
$$




$$
\begin{aligned}
& +\int_{0}^{t} \int_{\Omega} \alpha\left(\bar{u}^{N}\right) T_{k}\left(\bar{u}^{N}-\varphi\right)+\int_{0}^{t} \int_{\partial \Omega} \gamma\left(\bar{u}^{N}\right) T_{k}\left(\bar{u}^{N}-\varphi\right) \\
\leq & -\int_{0}^{t}\left\langle\frac{\partial \varphi}{\partial s}, T_{k}\left(u^{N}-\varphi\right)\right\rangle+\int_{\Omega} J_{k}\left(u^{N}(0)-\varphi(0)\right)-\int_{\Omega} J_{k}\left(u^{N}(t)-\varphi(t)\right) \\
& +\int_{0}^{t} \int_{\Omega} f_{N} T_{k}\left(\bar{u}^{N}-\varphi\right)+\int_{0}^{t} \int_{\partial \Omega} g_{N} T_{k}\left(\bar{u}^{N}-\varphi\right) \int_{0}^{t} \int_{\Omega} \frac{1}{p}\left|\Theta\left(T_{\bar{k}}\left(\bar{u}^{N}\right)\right)\right|^{p} \mathbf{1}_{Q(N, k)} .
\end{aligned}
$$

On the one hand, we have

$$
\begin{array}{cl}
\Theta\left(T_{\bar{k}}\left(u_{n}\right)\right) \rightarrow \Theta\left(T_{\bar{k}}(u)\right) & \text { strongly in } L^{p}\left(Q_{T}\right), \\
\left.D T_{\bar{k}}\left(\bar{u}^{N}\right) \rightarrow D T_{\bar{k}}(u)\right) & \text { weakly in } L^{p}\left(Q_{T}\right) .
\end{array}
$$

Thus,

$\Phi\left(D T_{\bar{k}}\left(\bar{u}^{N}\right)-\Theta\left(T_{\bar{k}}\left(\bar{u}^{N}\right)\right)\right) \rightarrow \Phi\left(D T_{\bar{k}}(u)-\Theta\left(T_{\bar{k}}(u)\right)\right) \quad$ weakly in $L^{p^{\prime}}\left(Q_{T}\right)$.

Now, as $D \varphi \mathbf{1}_{Q(N, k)}$ converges in $L^{p}\left(Q_{T}\right)$, we get

$$
\begin{aligned}
\int_{0}^{t} \int_{\Omega} \Phi\left(D T_{\bar{k}}\left(\bar{u}^{N}\right)-\Theta\left(T_{\bar{k}}\left(\bar{u}^{N}\right)\right)\right) & D \varphi \mathbf{1}_{Q(N, k)} \\
& \rightarrow \int_{0}^{t} \int_{\Omega} \Phi\left(D T_{\bar{k}}(u)-\Theta\left(T_{\bar{k}}(u)\right)\right) D \varphi \mathbf{1}_{Q(k)},
\end{aligned}
$$

where $Q(k)=\left\{\left|T_{\bar{k}}(u)-\varphi\right| \leq k\right\}$.

Using inequality 3.3 , we deduce that

$$
\left[\Phi\left(D T_{\bar{k}}\left(\bar{u}^{N}\right)-\Theta\left(T_{\bar{k}}\left(\bar{u}^{N}\right)\right)\right) D T_{\bar{k}}\left(\bar{u}^{N}\right)+\frac{1}{p}\left|\Theta\left(T_{\bar{k}}\left(\bar{u}^{N}\right)\right)\right|^{p}\right] \mathbf{1}_{Q(N, k)} \geq 0 .
$$

Therefore, by (5.15), 5.16) and Fatou's lemma,

$$
\begin{aligned}
& \int_{0}^{t} \int_{\Omega}\left[\Phi\left(D T_{\bar{k}}(u)-\Theta\left(T_{\bar{k}}(u)\right)\right) D T_{\bar{k}}(u)+\frac{1}{p}\left|\Theta\left(T_{\bar{k}}(u)\right)\right|^{p}\right] \mathbf{1}_{Q(k)} \\
& \leq \liminf \int_{0}^{t} \int_{\Omega}\left(\Phi\left(D T_{\bar{k}}\left(\bar{u}^{N}\right)-\Theta\left(T_{\bar{k}}\left(\bar{u}^{N}\right)\right)\right) D T_{\bar{k}}\left(\bar{u}^{N}\right)+\frac{1}{p}\left|\Theta\left(T_{\bar{k}}\left(\bar{u}^{N}\right)\right)\right|^{p}\right) \mathbf{1}_{Q(N, k)} .
\end{aligned}
$$

As, by hypothesis $\left(H_{3}\right)$, we have

$$
\left|\Theta\left(T_{\bar{k}}\left(\bar{u}^{N}\right)\right)\right|^{p} \leq(C \bar{k})^{p},
$$

this implies by (5.15) and the Dominated Convergence Theorem that

$$
\frac{1}{p} \int_{0}^{t} \int_{\Omega}\left|\Theta\left(T_{\bar{k}}\left(\bar{u}^{N}\right)\right)\right|^{p} \mathbf{1}_{Q(N, k)} \rightarrow \frac{1}{p} \int_{0}^{t} \int_{\Omega}\left|\Theta\left(T_{\bar{k}}(u)\right)\right|^{p} \mathbf{1}_{Q(k)} .
$$


By Lemma 5.4, we deduce that $u^{N}(t) \rightarrow u(t)$ in $L^{1}(\Omega)$ for all $t \in[0, T]$, which implies that

$$
\int_{\Omega} J_{k}\left(u^{N}(t)-\varphi(t)\right) \rightarrow \int_{\Omega} J_{k}(u(t)-\varphi(t)), \quad \forall t \in[0, T] .
$$

We follow the technique used in the proof of equality $(5.8)$ to show that

$$
\lim _{N \rightarrow \infty} \int_{0}^{t}\left\langle\frac{\partial u^{N}}{\partial s}, T_{k}\left(\bar{u}^{N}-\varphi\right)-T_{k}\left(u^{N}-\varphi\right)\right\rangle=0 .
$$

Finally, letting $N \rightarrow \infty$ and using the above results, the continuities of $\alpha$, $\gamma$ and the facts that

$$
\begin{aligned}
f_{N} & \rightarrow f & & \text { in } L^{1}\left(Q_{T}\right), \\
g_{N} & \rightarrow g & & \text { in } L^{1}\left(\Sigma_{T}\right), \\
T_{k}\left(\bar{u}^{N}-\varphi\right) & \rightarrow T_{k}(u-\varphi) & & \text { in } L^{\infty}\left(Q_{T}\right), \\
T_{k}\left(\bar{u}^{N}-\varphi\right) & \rightarrow T_{k}(u-\varphi) & & \text { in } L^{\infty}\left(\Sigma_{T}\right),
\end{aligned}
$$

we deduce that $u$ is an entropy solution of the nonlinear parabolic problem (1.1).

\section{References}

[1] A. Abassi and A. El Hachimi, Doubly nonlinear parabolic equations with absorbing terms as dynamical systems, Int. J. Math. Anal. 1 (2007), 1103-1124.

[2] A. Abassi, A. El Hachimi and A. Jamea, Entropy solutions to nonlinear Neumann problems with $L^{1}$-data, Int. J. Math. Statist. 2 (2008), 4-17.

[3] R. A. Adams, Sobolev Spaces, Academic Press, 1975.

[4] F. Andreu, N. Igbida, J. M. Mazón and J. Toledo, A degenerate elliptic-parabolic problem with nonlinear dynamical boundary conditions, Interfaces Free Bound. 8 (2006), 447-479.

[5] - - - - - , $L^{1}$ Existence and uniqueness results for quasi-linear elliptic equations with nonlinear boundary condition, Ann. Inst. H. Poincaré Anal. Non Linéaire 24, (2007), 61-89.

[6] F. Andreu, J. M. Mazón, S. Segura De León and J. Toledo, Existence and uniqueness for a degenerate parabolic equation with $L^{1}$-data, Trans. Amer. Math. Soc. 351 (1999), 285-306.

[7] - - - - - -, Quasi-linear elliptic and parabolic equations in $L^{1}$ with non-linear boundary conditions, Adv. Math. Sci. Appl. 7 (1997) 183-213.

[8] Ph. Bénilan, L. Boccardo, T. Gallouët, R. Gariepy, M. Pierre and J. L. Vázquez, An $L^{1}$ theory of existence and uniqueness of solutions of nonlinear elliptic equations, Ann. Scuola Norm. Sup. Pisa. 22 (1995), 241-273.

[9] F. Benzekri and A. El Hachimi, Doubly nonlinear parabolic equations related to the p-Laplacian operator: Semi-discretization, Electron. J. Differential Equations 2002, no. $113,14 \mathrm{pp}$.

[10] H. Brezis, Analyse fonctionnelle. Théorie et applications, Masson, Paris, 1983. 
[11] A. Dall'Aglio, Approximated solutions of equations with $L^{1}$ data. Application to the H-convergence of quasi-linear parabolic equations, Ann. Mat. Pura Appl. 170 (1996), $207-240$.

[12] J. I. Díaz, Nonlinear Partial Differential Equations and Free Boundaries, Vol. 1, Elliptic Equations, Res. Notes Math. 106, Pitman, London, 1985.

[13] J. I. Díaz and F. de Thélin, On a nonlinear parabolic problem arising in some models related to turbulent flows, SIAM J. Math. Anal. 25 (1994), 1085-1111.

[14] R. DiPerna and P.-L. Lions, On the Cauchy problem for the Boltzmann equation: global existence and weak stability, Ann. of Math. 130 (1989), 321-366.

[15] A. Eden, B. Michaux and J. M. Rakotoson, Semi-discretized nonlinear evolution equations as dynamical systems and error analysis, Indiana Univ. Math. J. 39 (1990), 737-783.

[16] G. Gagneux et M. Madaune-Tort, Analyse mathématique de modèles non linéaires de l'ingénierie pétrolière, Math. Appl. 22, Springer, 1996.

[17] A. El Hachimi and J. Igbida, Bounded weak solutions to nonlinear elliptic equations, Electron. J. Qualitat. Theory Differential Equations 2009, no. 10, 16 pp.

[18] A. El Hachimi and A. Jamea, Nonlinear parabolic problems with Neumann-type boundary conditions and $L^{1}$-data, ibid. 2007, no. 27, 22 pp.

[19] A. El Hachimi and M. R. Sidi Ammi, Thermistor problem: a nonlocal parabolic problem, Electron. J. Differential Equations 11 (2004), 117-128.

[20] D. Kinderlehrer and G. Stampacchia, An Introduction to Variational Inequalities and their Applications, Academic Press, 1980.

[21] D. Kröner and J.-F. Rodrigues, Global behaviour for bounded solutions of a porous media equation of elliptic-parabolic type, J. Math. Pures Appl. 64 (1985), 105-120.

[22] J. Liang and J.-F. Rodrigues, Quasilinear elliptic problems with nonmonotone discontinuities and measure data, Portugal. Math. 53 (1996), 239-252.

[23] J.-L. Lions, Quelques méthodes de résolution des problèmes aux limites non linéaires, Dunod, Paris, 1969.

[24] A. Prignet, Problèmes elliptiques et paraboliques dans un cadre non variationnel, thèse, 1996.

[25] T. Roubíček, Nonlinear heat equation with $L^{1}$-data, Nonlinear Differential Equations Appl. 5 (1998), 517-527.

[26] A. Siai, Nonlinear Neumann problems on bounded Lipschitz domains, Electron. J. Differential Equations 2005, no. 9, 16 pp.

[27] —, A fully nonlinear nonhomogeneous Neumann problem, Potential Anal. 24 (2006), $15-45$.

Abderrahmane El Hachimi, Jaouad Igbida, Ahmed Jamea

UFR Mathématiques Appliquées et Industrielles

Faculté des Sciences

B.P. 20, El Jadida, Morocco

E-mail: aelhachi@yahoo.fr

jigbida@yahoo.fr

a.jamea@yahoo.fr

Received on 4.1.2010;

revised version on 9.6.2010 